\title{
Association of adiponectin with mortality in older adults: the Health, Aging, and Body Composition Study
}

\author{
J. Poehls • C. L. Wassel • T. B. Harris • P. J. Havel • \\ M. M. Swarbrick • S. R. Cummings • A. B. Newman • \\ S. Satterfield • A. M. Kanaya • \\ for the Health ABC Study
}

Received: 12 November 2008 / Accepted: 29 December 2008 / Published online: 22 January 2009

(C) The Author(s) 2009. This article is published with open access at Springerlink.com

\begin{abstract}
Aims/hypothesis Despite inverse associations with insulin resistance and adiposity, adiponectin has been associated with both increased and decreased risk of cardiovascular disease. We examined whether adiponectin is associated with total and cardiovascular mortality in older adults with well-characterised body composition.

Methods We analysed data from 3,075 well-functioning adults aged 69-79 years at baseline. Mortality data were obtained over $6.6 \pm 1.6$ years. We used Cox proportional hazards models adjusting for covariates in stages to examine the association between adiponectin and total and cardiovascular mortality.

Results There were 679 deaths, $36 \%$ of which were from cardiovascular disease. Unadjusted levels of adiponectin were not associated with total or cardiovascular mortality.
\end{abstract}

\section{J. Poehls}

University of Wisconsin,

Madison, WI, USA

C. L. Wassel

University of California,

San Diego, CA, USA

T. B. Harris

Laboratory of Epidemiology, Demography and Biometry, IRP, National Institute on Aging,

National Institutes of Health,

Bethesda, MD, USA

\section{P. J. Havel}

Department of Molecular Biosciences,

School of Veterinary Medicine, University of California,

Davis, CA, USA

M. M. Swarbrick

Diabetes and Obesity Program,

Garvan Institute of Medical Research,

Sydney, Australia
However, after adjusting for sex and race, adiponectin was associated with an increased risk of both total mortality (hazard ratio $1.26,95 \%$ CI $1.15-1.37$, per SD) and cardiovascular mortality (hazard ratio 1.35 , 95\% CI 1.17 1.56 , per SD). Further adjustment for study site, smoking, hypertension, diabetes, prevalent heart disease, HDLcholesterol, LDL-cholesterol, renal function, fasting insulin, triacylglycerol, BMI, visceral fat, thigh intermuscular fat and thigh muscle area did not attenuate this association. This association between adiponectin and increased mortality risk did not vary by sex, race, body composition, diabetes, prevalent cardiovascular disease, smoking or weight loss.

Conclusions/interpretation Higher levels of adiponectin were associated with increased risks of total and cardiovascular mortality in this study of older persons.

\section{S. R. Cummings}

San Francisco Coordinating Center,

California Pacific Medical Center Research Institute,

San Francisco, CA, USA

S. R. Cummings $\cdot$ A. M. Kanaya $(\bowtie)$

UCSF Women's Health Clinical Research Center,

1635 Divisadero Street,

Suite 600, San Francisco,

CA 94115, USA

e-mail: Alka.Kanaya@ucsf.edu
A. B. Newman
University of Pittsburgh,
Pittsburgh,
PA, USA
S. Satterfield
University of Tennessee,
Memphis,
TN, USA 
Keywords Adiponectin · Adipose tissue .

Body composition $\cdot$ Epidemiology $\cdot$ Mortality

$\begin{array}{ll}\text { Abbreviations } & \\ \text { CVD } & \text { Cardiovascular disease } \\ \text { Health ABC } & \text { Health, Aging, and Body Composition } \\ \text { Study } & \text { Study }\end{array}$

\section{Introduction}

Adiponectin has been recognised as a biomarker of insulin resistance, lower levels being associated with higher BMI, reduced insulin sensitivity and less favourable lipid profiles [1]. Adiponectin also inhibits the development of atherosclerosis by downregulation of vascular adhesion factors, inhibition of foam cell formation and smooth muscle migration [2], and anti-inflammatory effects on macrophages [1]. These lines of evidence support the hypothesis that adiponectin may play a protective role in the development of cardiovascular disease.

However, human studies have shown inconsistent effects of adiponectin on cardiovascular disease (CVD) and mortality, possibly because of differences in sex, race/ ethnicity, age or body composition of study populations. A meta-analysis concluded that the association between adiponectin and CVD may not be as strong as previously considered [3].

We addressed these inconsistencies by analysing prospective data collected from older white and black men and women enrolled in the Health, Aging, and Body Composition (Health ABC) Study. We examined whether these associations were affected by well-characterised measures of body composition, and whether the associations differed by sex, race, smoking status, diabetes, prevalent CVD, body composition, weight loss and use of medications.

\section{Methods}

Participants in the Health, Aging, and Body Composition Study (Health ABC Study) were aged between 69 and 79 years at the baseline visit and were recruited between April 1997 and June 1998 from a random sample of Medicare beneficiaries from Pittsburgh (PA, USA) and Memphis (TN, USA). To be eligible, participants had to report no difficulty in walking $400 \mathrm{~m}$, climbing ten steps or performing basic activities of daily living. Individuals requiring assistive ambulatory devices, those with lifethreatening cancers and those planning to move were excluded. The study was approved by the institutional review boards of the University of California, San Francisco, the University of Pittsburgh and the University of Tennessee. All study participants provided informed consent.

Participants reported their racial group, age, sex, smoking history and comorbid conditions, including asthma/ chronic obstructive pulmonary disease, cancer, chronic kidney disease, congestive heart failure, CVD, diabetes, hypertension and stroke/transient ischaemic attack. Hypertension was defined as the use of an antihypertensive medication or systolic BP $>140 \mathrm{mmHg}$ or diastolic BP $>90 \mathrm{mmHg}$. Diabetes was defined by self-report, use of drugs to control diabetes, or fasting plasma glucose $\geq 6.9 \mathrm{mmol} / 1$ or $2 \mathrm{~h}$ post-challenge glucose $\geq 11.0 \mathrm{mmol} / \mathrm{l}$. Body composition (both regional adiposity and lean mass) was measured by computed tomography using standardised protocols as previously reported [4].

Participants underwent venipuncture after an overnight fast. Total adiponectin levels were assayed in 2002-2003 from frozen serum specimens; adiponectin was measured in duplicate by RIA (Linco, St Charles, MO, USA) with an intra-assay coefficient of variation of 1.8-3.6\%.

Mortality outcomes were assessed until 31 August 2005. Deaths were adjudicated by a central committee for immediate and underlying causes of death using established criteria, including review of death certificate, all recent hospital records, and interview with the next of kin. Cardiovascular mortality was defined as atherosclerotic cardiovascular disease (definite fatal myocardial infarction, or definite or possible fatal CHD), stroke, atherosclerotic disease other than coronary or cerebrovascular, and other CVD.

Associations of baseline covariates with total and CVD mortality were assessed using Cox models. We rescaled body composition measures by their standard deviations and assessed correlations among the body composition variables to identify and eliminate collinearity. We used multivariable Cox proportional hazards models to examine associations between adiponectin and total mortality and mortality from CVD. In nested sequences of models using only those with all covariates $(n=2,548)$, we adjusted for age, sex, race, site, smoking, hypertension, diabetes, CHD, LDL-cholesterol and estimated GFR. Then, we adjusted our models for body composition, including BMI, abdominal visceral fat, thigh intermuscular fat and thigh muscle area. Last, models were adjusted for HDL-cholesterol, insulin and triacylglycerol.

We checked whether sex, race, diabetes, CVD, smoking status, body composition, weight loss and use of medications may modify the association between adiponectin and mortality. Participants taking a medication known to increase adiponectin (insulin, thiazolidinedione, angiotensin receptor blockers, angiotensin-converting enzyme inhibitors, beta blockers, and/or statins) were compared with 
Table 1 Association between adiponectin and total and cardiovascular mortality with sequential adjustment for explanatory variables in staged models

\begin{tabular}{lrr}
\hline Model & Total mortality & CVD mortality $^{\mathrm{a}}$ \\
\hline Unadjusted adiponectin per SD $^{\mathrm{b}}$ & $1.07(0.98-1.16)$ & $1.14(0.99-1.31)$ \\
Adiponectin adjusted for age and sex & $1.16(1.06-1.26)$ & $1.23(1.06-1.42)$ \\
Model $1^{\mathrm{c}}$ & $1.24(1.13-1.36)$ & $1.36(1.18-1.57)$ \\
Model $^{\mathrm{c}}$ & $1.22(1.11-1.35)$ & $1.36(1.16-1.59)$ \\
Model $^{\mathrm{c}}$ & $1.27(1.14-1.40)$ & $1.38(1.17-1.64)$ \\
\hline
\end{tabular}

Data are HR $(95 \% \mathrm{CI})$

${ }^{a}$ Cardiovascular mortality includes all CHD, all stroke and all CVD deaths combined

${ }^{\mathrm{b}}$ Analysed per SD $(6.87 \mu \mathrm{g} / \mathrm{ml})$ increase in adiponectin

${ }^{c}$ Model 1 was adjusted for age, sex, race, site, smoking, hypertension, diabetes, prevalent CHD, LDL-cholesterol, estimated GFR (per SD) and current oral oestrogen use; model 2 was model 1 with adjustment for BMI, abdominal visceral fat, thigh intermuscular fat and thigh muscle area (all per SD); model 3 was model 2 with adjustment for HDL-cholesterol, insulin and triacylglycerol (all natural log transformed)

those not using any of these medications. We also evaluated adiponectin's association with mortality per year and by tertile of follow-up time.

The proportional hazards assumption was verified by examining plots of Schoenfeld residuals vs transformed time and assessing interactions of the all predictors with log transformed time. We used SAS Version 9.1 (SAS Institute, Cary, NC, USA) and SPlus Version 6.1 (Insightful, Seattle, WA, USA).

\section{Results}

The average age of the 3,075 participants was $73.6 \pm 2.9$ years. There were 679 (22.1\%) deaths, of which 247 (36.4\%) were from CVD after a mean $6.6 \pm 1.6$ years of follow-up. Mean adiponectin concentration was higher in women than in men $(13.3 \pm 7.4 \mathrm{vs} 9.5 \pm 5.6 \mu \mathrm{g} / \mathrm{ml}, p<0.001)$ and in whites than in blacks $(12.8 \pm 7.0$ vs $9.5 \pm 6.3 \mu \mathrm{g} / \mathrm{ml}, p<0.001)$. Lower adiponectin levels were associated with higher levels of BMI, abdominal visceral fat, thigh intermuscular fat and thigh muscle area. Higher adiponectin concentrations were associated with older age, female sex and chronic kidney disease $(n=653)$.

Table 1 shows the association between adiponectin and total and cardiovascular mortality with sequential adjustment. Unadjusted adiponectin concentration showed a trend towards increased total mortality risk (HR 1.07, 95\% CI 0.98-1.16) and cardiovascular mortality risk (HR $1.14,95 \%$ CI $0.99-1.31)$. After adjustment for only sex or race, adiponectin was significantly associated with increased risk of both mortality outcomes and remained robustly associated with increased risk of total mortality (HR 1.27, 1.14-1.40, per SD) and CVD mortality (HR $1.38,1.17-1.64$, per SD) in models fully adjusted for body composition, comorbid diseases and metabolic factors.
We evaluated subgroups between which adiponectin levels may be expected to vary (Fig. 1). Those with overweight BMI had increased risk of CVD mortality ( $p$ for interaction 0.03 ). The association between adiponectin and mortality did not vary among those with higher or lower levels of regional adiposity or lean muscle mass. We examined two weight loss subgroups: (1) weight loss $>5 \%$ from baseline during followup vs stable weight or weight gain; and (2) weight loss between age 50 and the baseline visit. We found no evidence of interaction between adiponectin and prior or prospective weight loss for either outcome. Among 882 (35\%) participants taking one or more of the medications that may affect adiponectin, concentrations were higher among medication users than among non-users $(12.1 \pm 6.9$ vs $11.2 \pm 6.7 \mu \mathrm{g} / \mathrm{ml}$, $p<0.001)$. Medication users had higher risk of total mortality with increasing adiponectin than non-users ( $p$ for interaction, 0.02 ), but there was no difference according to medication use for CVD mortality ( $p$ for interaction, 0.85). This increased risk of adiponectin with mortality did not vary with follow-up time.

\section{Discussion}

In a cohort of elderly black and white Americans, adiponectin was significantly associated with increased risk of both total and cardiovascular mortality after adjusting only for sex or race. This association was not diminished after adjusting for other covariates, including body composition and other variables associated with the metabolic syndrome. The association was robust and did not vary with sex, race, smoking, diabetes, CVD, body composition, weight loss, medication use or length of follow-up.

Once adjusted for either sex or race, the results of our study are consistent with other published studies showing that higher adiponectin levels are associated with increased cardiovascular mortality and all-cause mortality $[5,6]$. 


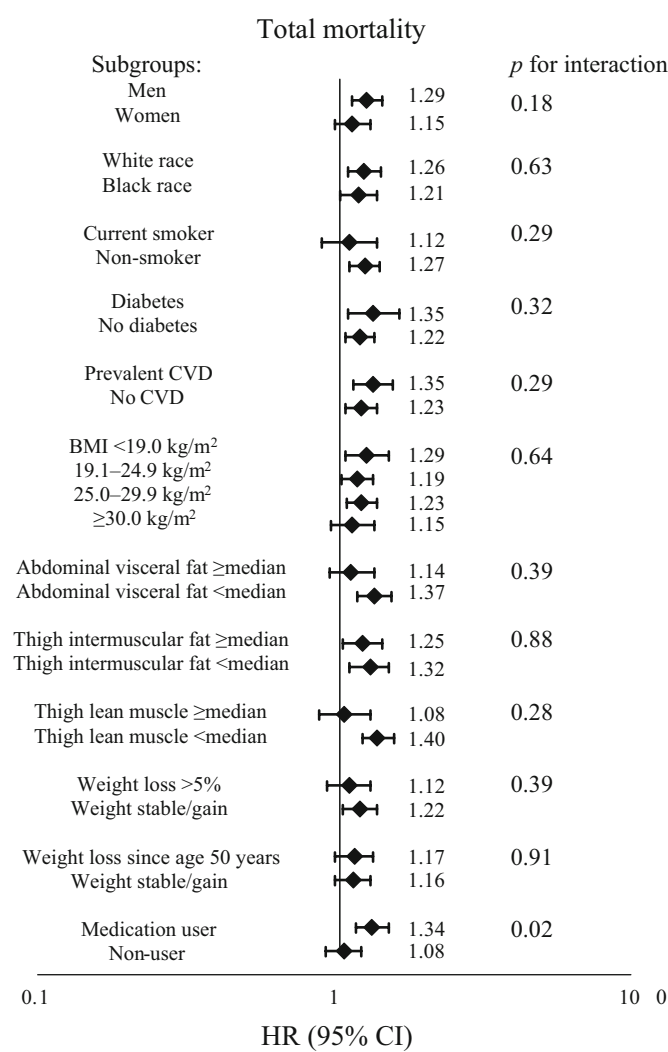

Fig. 1 Association between adiponectin (per SD) and total and cardiovascular mortality by subgroup after adjustment for age, sex, race, study site, smoking, hypertension, diabetes, prevalent CVD, LDL-cholesterol, estimated GFR, oestrogen use, BMI, abdominal visceral fat, thigh intermuscular fat, thigh muscle area, HDLcholesterol, insulin and triacylglycerol (without adjustment for the subgroup variable of interest). The $p$ value for interaction is shown for each subgroup. Median for abdominal visceral fat was $140.9 \mathrm{~cm}^{2}$ for

However, our results contradict studies that have reported that higher adiponectin levels are cardioprotective [7-9]. We expected that differences in body composition would explain the discrepancy in the literature. While each measure we examined was associated with adiponectin and mortality univariately, these body composition measures did not explain or modify the positive association between adiponectin and mortality. Another explanation may be that overweight people surviving to old age may have characteristics that protect them from adverse effects of overweight. Weight loss is associated with increased adiponectin levels, and higher adiponectin levels in the elderly may be a marker for a catabolic process causing weight loss. However, participants who lost $>5 \%$ of body weight had an increase in their risk of mortality similar to that of those with stable weight or weight gain. Another possible explanation is that most studies measure total adiponectin, not its high molecular weight (HMW) complex. A higher ratio of HMW to total adiponectin was best correlated with enhanced insulin sensitivity [1] and varies
Cardiovascular mortality

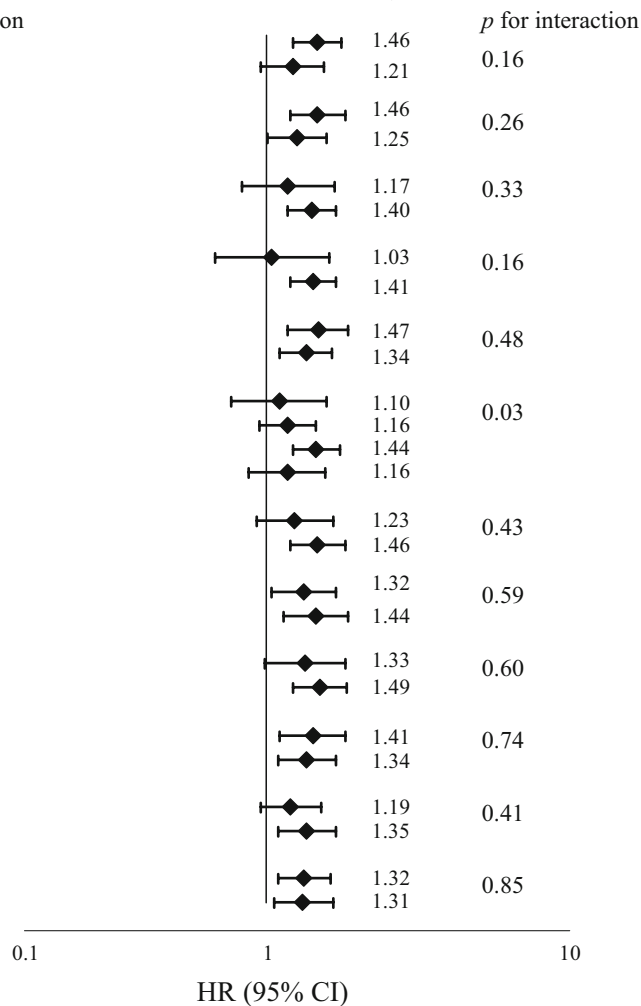

men and $119.7 \mathrm{~cm}^{2}$ for women. Median thigh intermuscular fat was $8.45 \mathrm{~cm}^{2}$ for men and $9.32 \mathrm{~cm}^{2}$ for women. Median thigh lean muscle was $130.5 \mathrm{~cm}^{2}$ for men and $90.7 \mathrm{~cm}^{2}$ for women. 'Medication user' refers to any participant taking a medication that increases adiponectin concentrations: insulin, a thiazolidinedione, an ACE inhibitor, an angiotensin II receptor blocker, a beta blocker, or a statin medication. 'Non-user' refers to those not on any of these medications

by age, sex and race. However, a recent study found no association between HMW adiponectin or the ratio of HMW/total adiponectin and incident CHD [10]. We are not aware of any studies that have examined the association between HMW adiponectin and mortality.

Since the Health ABC Study cohort comprises community-dwelling older adults without advanced physical or cognitive impairment, our findings may not generalise to younger populations or the frail elderly. Unaccounted associated factors may influence the association of adiponectin with mortality. We had only one baseline measure of adiponectin, and this snapshot in time may fail to capture the complexity of the compensatory mechanisms involved in regulating adiponectin concentrations prior to death.

In this prospective analysis of elderly black and white men and women, adiponectin was associated with increased total and cardiovascular mortality. Body composition, comorbid diseases, metabolic covariates, weight loss and medication use did not explain this increased risk of mortality. Differences in demographic characteristics be- 
tween the different populations studied may in part explain previous inconsistencies in the associations of adiponectin with cardiovascular and total mortality.

Acknowledgements A.M. Kanaya was funded by the National Institutes of Health, grants \#K23HL080026 and \#R21DK068608. The Health ABC Study was funded through contracts with National Institute on Aging (NIA) contracts N01-AG-6-2101, N01-AG-6-2103 and N01AG-6-2106. This research was supported in part by the Intramural Research Program of the NIH, NIA and included substantial involvement of NIA staff in data collection, analysis, interpretation and review, and approval of the manuscript. A. M. Kanaya had full access to all the data in the study and takes responsibility for the integrity of the data and the accuracy of the data analysis.

Duality of interest statement The authors declare that there is no duality of interest associated with this manuscript.

Open Access This article is distributed under the terms of the Creative Commons Attribution Noncommercial License which permits any noncommercial use, distribution, and reproduction in any medium, provided the original author(s) and source are credited.

\section{References}

1. Trujillo ME, Scherer PE (2005) Adiponectin-journey from an adipocyte secretory protein to biomarker of the metabolic syndrome. J Intern Med 257:167-175
2. Ouchi N, Kihara S, Arita Y et al (2001) Adipocyte-derived plasma protein, adiponectin, suppresses lipid accumulation and class A scavenger receptor expression in human monocyte-derived macrophages. Circulation 103:1057-1063

3. Sattar N, Wannamethee G, Sarwar N et al (2006) Adiponectin and coronary heart disease: a prospective study and meta-analysis. Circulation 114:623-629

4. Goodpaster BH, Krishnaswami S, Resnick H et al (2003) Association between regional adipose tissue distribution and both type 2 diabetes and impaired glucose tolerance in elderly men and women. Diabetes Care 26:372-379

5. Laughlin GA, Barrett-Connor E, May S, Langenberg C (2007) Association of adiponectin with coronary heart disease and mortality: the Rancho Bernardo study. Am J Epidemiol 165:164-174

6. Wannamethee SG, Tchernova J, Whincup P et al (2007) Associations of adiponectin with metabolic and vascular risk parameters in the British Regional Heart Study reveal stronger links to insulin resistance-related than to coronary heart disease risk-related parameters. Int J Obes (Lond) 31:1089-1098

7. Pischon T, Girman CJ, Hotamisligil GS, Rifai N, Hu FB, Rimm EB (2004) Plasma adiponectin levels and risk of myocardial infarction in men. JAMA 291:1730-1737

8. Kumada M, Kihara S, Sumitsuji S et al (2003) Association of hypoadiponectinemia with coronary artery disease in men. Arterioscler Thromb Vasc Biol 23:85-89

9. Frystyk J, Berne C, Berglund L, Jensevik K, Flyvbjerg A, Zethelius B (2007) Serum adiponectin is a predictor of coronary heart disease: a population-based 10-year follow-up study in elderly men. J Clin Endocrinol Metab 92:571-576

10. Sattar N, Watt P, Cherry L, Ebrahim S, Davey Smith G, Lawlor DA (2008) High molecular weight adiponectin is not associated with incident coronary heart disease in older women: a nested prospective case control study. J Clin Endocrinol Metab 93:1846-1849 